\title{
Transport of water-glycerol mixtures in porous materials studied with NMR imaging
}

\author{
C. J. Kuijpers ${ }^{1,2}$, H. P. Huinink ${ }^{2}$, O. C. G. Adan ${ }^{2,3}$, \\ S. J. F. Erich ${ }^{2,3} \&$ N. Tomozeiu ${ }^{4}$ \\ ${ }^{I}$ Materials Innovations Institute (M2i), The Netherlands \\ ${ }^{2}$ Applied Physics Department, Eindhoven University of Technology, \\ The Netherlands \\ ${ }^{3}$ Organisation of Applied Scientific Research, TNO, The Netherlands \\ ${ }^{4} R \& D$ Department, Océ Technology BV, The Netherlands
}

\begin{abstract}
The penetration of water-glycerol mixtures in a porous material is studied by using NMR (Nuclear Magnetic Resonance) measurements in view of understanding and improving water based inks performance on paper. $\mathrm{Al}_{2} \mathrm{O}_{3}$ samples are used as well-defined model systems to quantify the penetration of each mixture component. We investigated the dependence of glycerol concentration in water on the penetration speed, and quantify to what extend the mixture separates in the porous material. We visualized water and glycerol as a function of time and link the behavior of this mixture to the rheological properties. Finally, we investigated the effect of pore size on the imbibition behavior.

We found that water-glycerol mixtures penetrate the porous material as a single liquid in both $200 \mathrm{~nm}$ and $1100 \mathrm{~nm}$ porous $\mathrm{Al}_{2} \mathrm{O}_{3}$. Furthermore we showed that the imbibition process can be described by a modified version of Washburn's equation and that the behavior can be scaled with liquid and media parameters to result in one generic curve. This implies that the complex mixture behaves as a homogeneous liquid and the sorption behavior is determined by the average viscosity and surface tension of the mixture.
\end{abstract}

Keywords: NMR imaging, complex mixtures, imbibition, porous media, Washburn's equation, paper. 


\section{Introduction}

Research on porous media, and more specifically, liquid sorption in porous media has been performed for over a century with pioneers like Darcy [1], Washburn [3] and Richards [2], all starting their research in the late $19^{\text {th }}$ and early $20^{\text {th }}$ century. Their research is still valuable and their equations still form the bases for the current analytical and numerical models that describe liquid penetration in porous materials.

Over the years, the complexity and accuracy of the models has increased due to the interest of the industry on this fascinating group of materials. Especially in the field of (microbial) enhanced oil recovery (Sarkar et al. [4]), $\mathrm{CO}_{2}$ storage [5, 6] and membrane fuel cell technology (Wang et al. [7]), understanding the process of liquid penetration in porous media is necessary to keep improving the current techniques and to remain economically and environmentally feasible in the future.

The latest models are capable of describing deformable porous media [8-10], multiphase flow [11, 12], wettability influences (Blunt [13]) and particle containing flows $[14,15]$. An overview of the existing multi-scale and multiphysics methods is given by Helmig et al. [16]. Although current research focusses on modelling of liquid sorption in porous media, experimental data is required to provide to validate these models for real life applications.

Experimental data exists for techniques ranging from optical techniques on transparent samples [17-19] and micro-models (Stevar and Vorobev [20]) to averaging methods on sand columns, packed beads [21] and 'real' materials such as bricks (Pel et al. [22]), and thin films (Reuvers et al. [23]). However, experimental data describing the dynamics of liquid penetration in paper is scarce.

The paper industry existed far before Darcy, Washburn and Richards did their research, with paper existing for thousands of years and the first printing press invented in the 14th century. Although paper is in existence for a long period of time, the printing industry is still developing as a result of the economic and environmental pressure to manufacture cleaner inks. In the printing industry, the search to reduce the volatile components in ink has led to the development of water-based inks. Water-based inks are complex mixtures containing, among others, water, co-solvent (e.g. glycerol, ethanol or isopropanol) and particles (e.g. pigment). In order to improve present water based inks, we need to understand the imbibition of these complex mixtures in porous materials in general and paper in particular.

The penetration of the fluids and particles in the paper influences the quality of the printed image. In order to better understand the process of forming a polymeric film on paper, we focus on the four dominating processes in paper-ink interaction which are schematically drawn in fig. 1. These include capillary suction, evaporation, sorption into the paper fibres and agglomeration of the pigment particles. The focus of this research will be on capillary suction of liquid mixtures. Due to the complexity of paper, $\mathrm{Al}_{2} \mathrm{O}_{3}$ cylinders are used as a simple porous material to understand the key processes in real printing paper. To measure the imbibition of liquid mixtures in these $\mathrm{Al}_{2} \mathrm{O}_{3}$ samples, Nuclear Magnetic Resonance (NMR) is used. NMR [22-24] is a non-destructive method that can be 
used to determine the position of specific atoms which can be used to visualise the liquids in the porous samples.
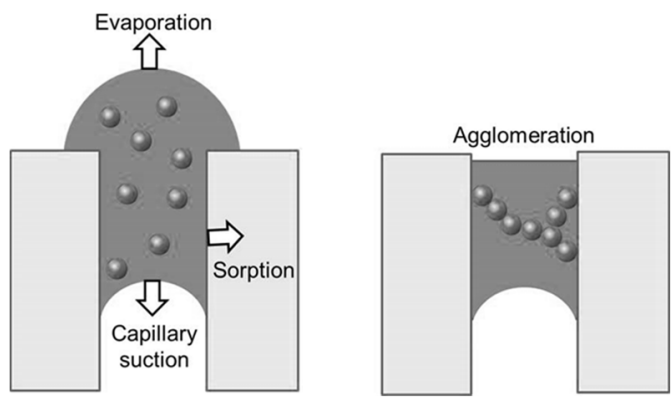

Figure 1: The four dominant processes in ink penetration in paper.

Gombia and Bortolotti [25] used NMR to validate models of water imbibition in treated and untreated porous media but NMR can also be used to look at colloidal deposition in a porous medium, as shown by Fridjonsson et al. [26]. They used NMR to investigate the spatial deposition of particles in a colloidal suspension when penetrating in a porous material.

Summarizing, the behavior of liquid sorption in porous media has been the subject of study for over a century. However, especially in cases when looking at (very) complex liquid mixtures or complex porous materials, our understanding is still inadequate. In this study we aim to understand the imbibition of liquid mixtures consisting of water and glycerol in a porous material. This research focusses at understanding how far and how fast each component penetrates.

\section{Theory}

Since the focus of this work is on capillary suction, we consider one of the simplest cases describing imbibition of a mixture in a porous material. Most porous media can be approximated by an array of cylinders, for which the Bell-Cameron-LucasWashburn equation, commonly referred to as Washburn's equation, is valid. This equation uses the next basic assumptions (Bell and Cameron [27]):

1. The imbibing mixture can be regarded as a homogeneous liquid with one (constant) viscosity.

2. The cylinders are considered straight without branches.

3. The Reynolds number is considered low (laminar flow).

4. Dynamic effects in surface tension and capillary pressure can be neglected. Washburn's equation (eqn (1)), describes the flow in a bundle of fully wetting capillary tubes (Washburn [3]).

$$
L^{2}=\frac{\gamma D t}{4 \eta}
$$

wherein $D$ is the average diameter of the capillary, $\gamma$ the surface tension of the penetrating liquid, $\eta$ the dynamic viscosity and $t$ the time it takes the liquid to penetrate over a length $L$ into the porous material. 
This equation can be modified for capillaries that are not perfectly wetting (non-zero contact angle) by including the contact angle $\theta$ for the liquid in this capillary. By adding the tortuosity $\phi$ one can make the equation applicable to 'real' porous media. The tortuosity corrects for the longer path length the liquid has to take when imbibing into the porous material. This results in:

$$
L^{2}=\frac{\gamma \cos (\theta) D t}{4 \eta \phi^{2}}
$$

\section{Materials and methods}

\subsection{NMR imaging}

NMR is a method which probes molecular properties by measuring the interaction of atomic nuclei with external and static magnetic fields and radio-frequency radiation. Basically NMR is the r.f. communication between the laboratory electronics such as transmitters and receivers on the one hand, and the magnetic polarization of the atomic nuclei on the other (Blümich [28]). The frequency with which the atomic nuclei respond is called the Larmor frequency, which is related to the strength of the magnetic field $\boldsymbol{B}$ and is given by.

$$
\omega_{L}=-\gamma|\boldsymbol{B}|
$$

The gyromagnetic ratio $\gamma$ is a constant depending on the type of nucleus. In order to use NMR imaging, inhomogeneous magnetic fields are used to make the atoms at a specific location resonate. This makes $2 \mathrm{D}$ or even $3 \mathrm{D}$ images of a liquid in a porous medium possible. The position dependent magnetic field is achieved by applying a magnetic field gradient $\boldsymbol{G}_{\boldsymbol{x}}$.

$$
\boldsymbol{B}=\boldsymbol{B}_{\boldsymbol{o}}+\boldsymbol{G}_{\boldsymbol{x}} \boldsymbol{x}
$$

Magnetic field inhomogeneities (either by a gradient or magnet inhomogeneities) causes dephasing, this is reversible as was discovered by Hahn [29], and a rephasing pulse can be applied to rephase the spins. This is now known as the Hahn spin echo sequence. The measured signal is proportional to the amount of excited atoms and therefore to the amount of liquid. A more detailed description of NMR principles and pulse sequences can be found in Blümich [28] and Callaghan [30].

\subsection{Samples and mixtures}

In this research, we use $\mathrm{Al}_{2} \mathrm{O}_{3}$ cylinders as a first model approximation for printing paper. The cylinders are $20 \mathrm{~mm}$ in diameter and $50 \mathrm{~mm}$ in length with a welldefined pore size distribution. We used $\mathrm{Al}_{2} \mathrm{O}_{3}$ cylinders with an average pore diameter of $200 \mathrm{~nm}$ which is similar to the pore size generally found in the coating of printing papers. The cylinders with an average pore diameter of $1100 \mathrm{~nm}$ act as a representation of the pores in the bulk of the paper. Note that a pigment particle used in water-based inks is typically about $60-100 \mathrm{~nm}$. The pore size distribution is measured by mercury intrusion porosimetry (MIP).

The liquids used in this research are demineralised water, glycerol (min. $99.0 \%$ ) and a mixture of both. The mixtures were defined by weight using a lab 
scale (Mettler Toledo PG2002-S) with an accuracy of $0.01 \mathrm{~g}$. Glycerol is used as an example of a co-solvent.

\subsection{Experimental set-up}

The NMR measurements were performed with a home built NMR scanner which has the possibility to measure hydrogen profiles (Kopinga and Pel [31]). The magnetic field of the scanner is generated by a water-cooled iron cored electromagnet resulting in a $\boldsymbol{B}_{\boldsymbol{o}}$ field of $0.7 \mathrm{~T}$. Furthermore gradients of $0.45 \mathrm{~T} / \mathrm{m}$ can be applied to measure the hydrogen concentration at a specific position. A step motor was used to position our sample holder through the sensitive part of our setup to measure the whole sample. In our measurements we used 4 averages at each position and an echo time of $700 \mathrm{~ms}$. This resulted in a measurement time of 10 minutes for the whole sample. Fig. 2 gives a picture of our set-up as well as a schematic drawing. Apart from the coils an important part of the set-up is the insert. The insert contains a tuned r.f. coil to send and receive signals and a faraday cage to reduce the noise.
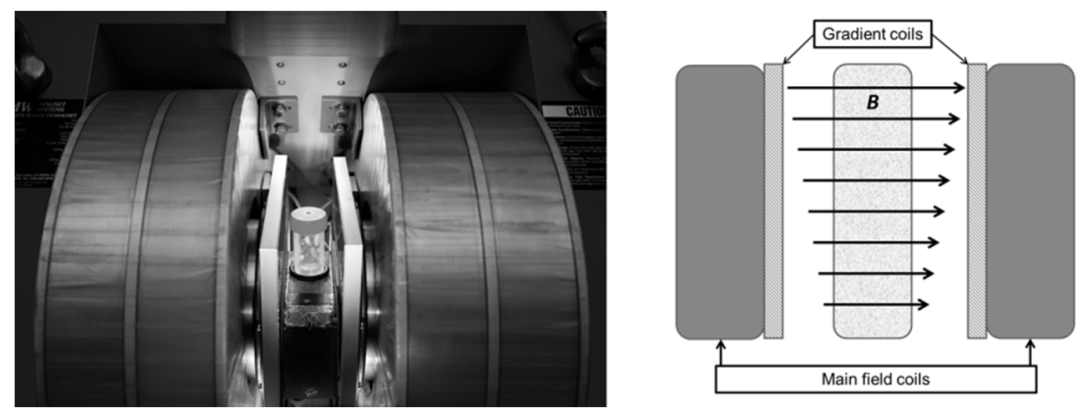

Figure 2: Picture of the experimental set-up (left) and schematic drawing of the set-up (front view) including the magnetic field (right).

The sample holder used in our experiments is made of PVC and consists of two parts. The sample holder is schematically drawn in fig. 3 . The bottom part of the sample holder contains a reference liquid that is separated from the actual measurement area. The reference liquid used in this study is $\mathrm{CuSO}_{4} 0.01 \mathrm{M}$. This mixture gives short relaxation times, which ensures that short measurement times are possible. It is used to verify that the measured signal does not change over time due to variations of the environment or the NMR electronics.

The measurement compartment holds the liquid below the $\mathrm{Al}_{2} \mathrm{O}_{3}$ samples to exclude air trapping in the samples. Another advantage of having the liquid below the sample is to prevent leaking from the sides of the sample. This ensures all the liquid penetrates the sample from the bottom. The liquid is fed to the measurement compartment by two peristaltic pumps (Lead Fluid BQ50s). At all times the flow through the outlet tube is kept higher $(30 \mathrm{rpm})$ than the flow in the inlet tube $(5$ $\mathrm{rpm}$ ) to ensure a constant liquid level. The connecting tubes have an inner diameter of $2.4 \mathrm{~mm}$ and a wall thickness of $0.8 \mathrm{~mm}$. 


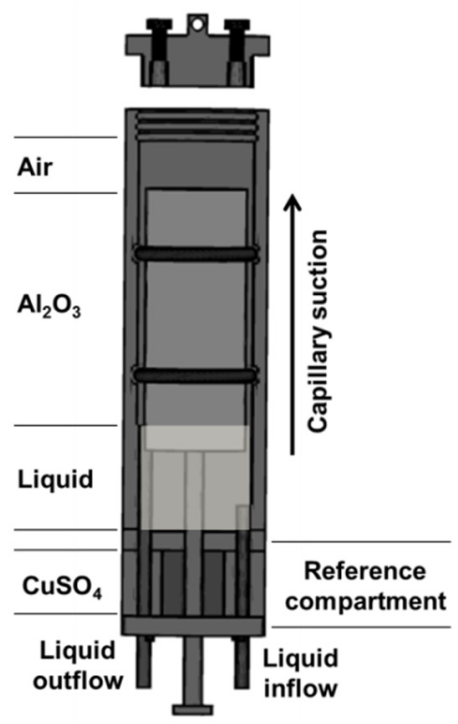

Figure 3: Sample holder used for the NMR experiments with a reference compartment and a measurement compartment containing the liquid and the porous $\mathrm{Al}_{2} \mathrm{O}_{3}$ sample.

\section{Experiments}

We conducted four sets of experiments with the porous $\mathrm{Al}_{2} \mathrm{O}_{3}$ samples. We measured the penetration of water and water-glycerol (50 wt \%) in the 200 and $1100 \mathrm{~nm}$ porous samples. However, the penetration of water in the $1100 \mathrm{~nm}$ porous $\mathrm{Al}_{2} \mathrm{O}_{3}$ samples was very fast so that only one profile could be recorded before the sample was fully saturated. The results from this measurement are therefore excluded.

\subsection{Water sorption in $200 \mathrm{~nm} \mathrm{Al}_{2} \mathrm{O}_{3}$}

We started with measurements of water penetrating into $200 \mathrm{~nm}$ porous $\mathrm{Al}_{2} \mathrm{O}_{3}$. The resulting signal profiles as a function of time are given in fig. 4 . It takes 10.5 minutes to record a signal profile of the entire sample and reference. The signal intensity is related to the moisture content in the sample. The higher the signal, the higher the number of hydrogen atoms in the measurement domain, which means more liquid at this position.

In order to compare the found signal profiles with theoretically expected behavior, we determined the position of the wetting front. This is defined as the position, where the height of the moisture profile is half that of the saturation value. Plotting this position as a function of time, gives a basis for comparison of the experimental values to the modified version of Washburn's equation as given in eqn (2). 


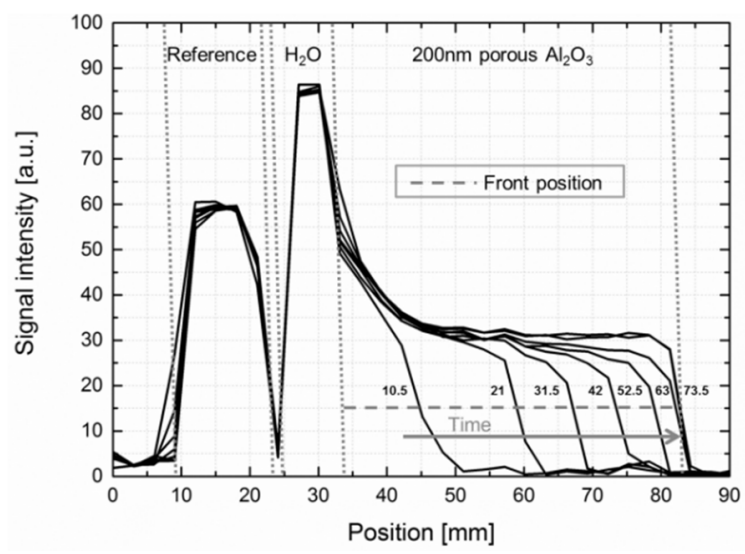

Figure 4: The measured signal profiles for a water uptake experiment in $200 \mathrm{~nm}$ porous $\mathrm{Al}_{2} \mathrm{O}_{3}$. Each profile takes 10.5 minutes, so it takes water about 70 minutes to fully saturate the sample.

To do this, the properties of the liquid ( $\gamma$ and $\eta$ ) need to be known as well as the properties of the sample $(D, \phi$ and $\theta)$. The liquid parameters can be found in the literature and the average pore diameter of the sample can be determined by MIP measurements. However, the contact angle of water on porous $\mathrm{Al}_{2} \mathrm{O}_{3}$ is not available and, up to this point, the tortuosity of the samples is not measured yet. Therefore we used the tortuosity and the contact angle as a fitting parameter, to check whether the speed of the wetting front can be captured by the modified Washburn equation. The experimental data and the theoretical prediction are given in fig. 5. We measured 3 samples with the same dimension and average pore size in three subsequent measurements.

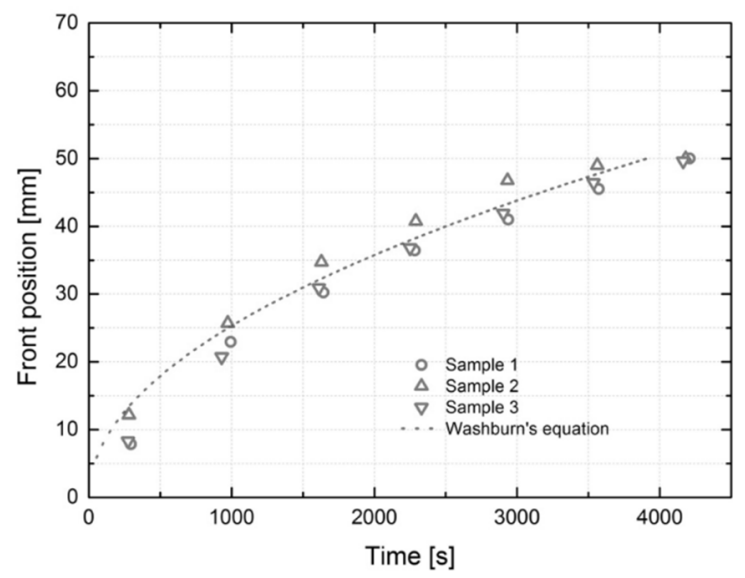

Figure 5: Comparison between experimental data and theoretical prediction for water penetration in $200 \mathrm{~nm}$ porous $\mathrm{Al}_{2} \mathrm{O}_{3}$. 
From fig. 5 we can see that the modified Washburn's equation captures the phenomena adequately in the case that the fitting parameter $\cos (\theta) / \phi^{2}=0.17$. When we assume perfect wetting $(\theta=0)$, this appears a reasonable value since this gives a tortuosity of about 2.4. Generally the tortuosity of a porous medium is between 1 and 10 .

\subsection{Water-glycerol (50 wt \%) sorption in $200 \mathrm{~nm}$ porous $\mathrm{Al}_{2} \mathrm{O}_{3}$}

The next step towards water based inks is the inclusion of co-solvent in the imbibing liquid. We therefore moved to experiments with a water-glycerol mixture as the absorbing liquid, wherein the glycerol content is $50 \mathrm{wt} \%$. We followed the same procedure as in the water case and plotted the front position as a function of time (fig. 6).

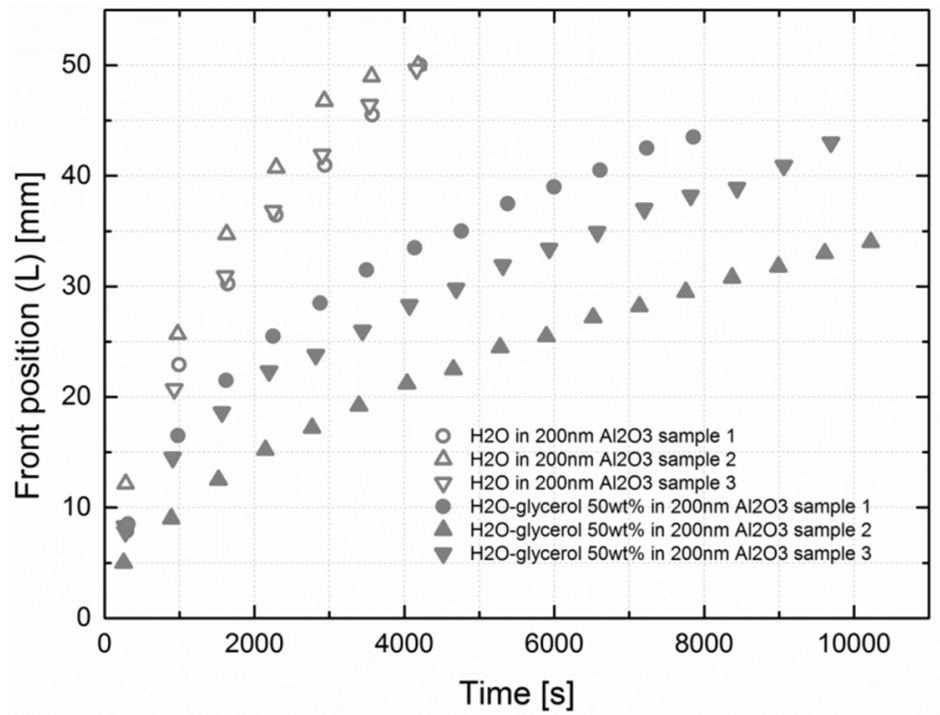

Figure 6: Front position as function of time for water and water-glycerol sorption in $200 \mathrm{~nm}$ porous $\mathrm{Al}_{2} \mathrm{O}_{3}$.

From fig. 6 we conclude that the sorption of the water-glycerol mixture is significantly slower than the sorption of pure water in the same material which is expected due to the higher viscosity. Furthermore, there is a significant variation for the front speed of the 3 water-glycerol measurements. The origin of this variation is still unknown. Note that this variation was not visible when the same samples were used in the water sorption experiments.

We rescaled the graph of fig. 6 with the liquid parameters $\gamma$ and $\eta$ and the average pore size $D$ to investigate if there is a fundamental difference between the sorption of water and the sorption of the $50 \mathrm{wt} \%$ water-glycerol mixture. The parameters used for this rescaling are given in table 1 and the resulting graph is given in fig. 7. 
Table 1: Liquid properties of water and water-glycerol mixture.

\begin{tabular}{|l|c|c|c|}
\hline & Water & Glycerol & $\begin{array}{c}50 \text { wt \% } \\
\text { Water-Glycerol }\end{array}$ \\
\hline Density (kg/l) [32] & 0.998 & 1.261 & 1.125 \\
\hline Viscosity (mPa*s) [33] & 1.005 & 1410 & 6.00 \\
\hline Surface tension (mN/m) [34] & 71.68 & 63.4 & 68.7 \\
\hline
\end{tabular}

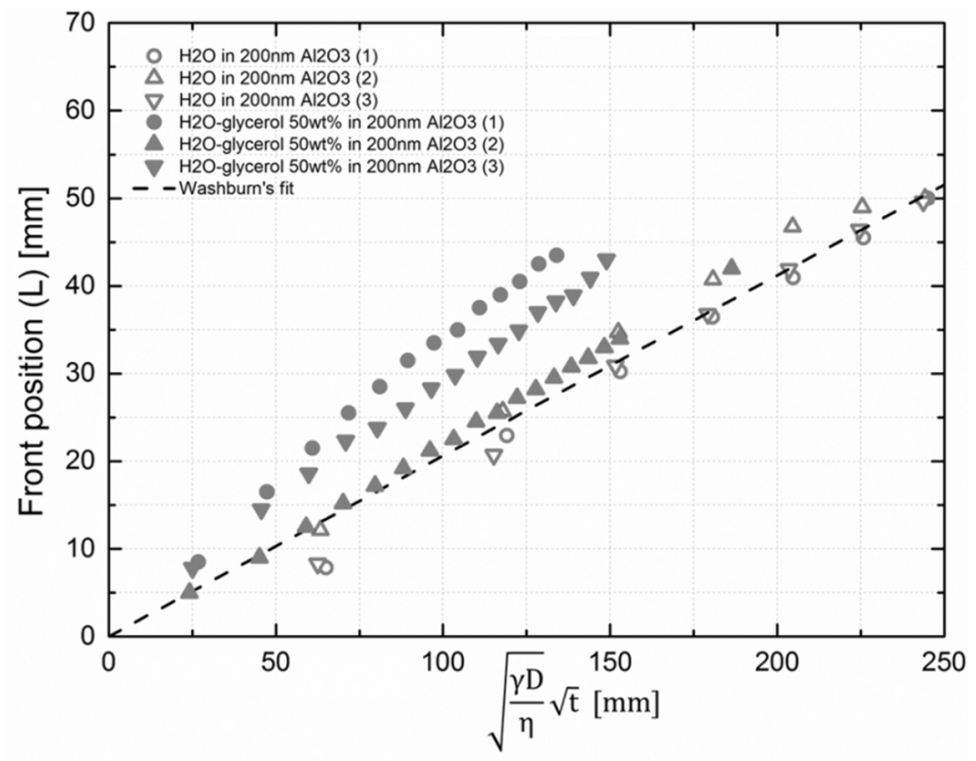

Figure 7: Front position versus rescaled time for the sorption of water and water-glycerol mixture in $200 \mathrm{~nm}$ porous $\mathrm{Al}_{2} \mathrm{O}_{3}$.

Fig. 7 indicates that the sorption of the $50 \mathrm{wt} \%$ water-glycerol mixture can be governed by similar equations as in the water sorption case when looking at sample 2. However, sample 1 and 3 show different behavior with a faster uptake rate. This could indicate a problem with the mixture (since a lower glycerol concentration would result in faster uptake) but up to now we have not found the origin of this variation. We do not know if this is a real effect or a measurement error which is being investigated.

In the case of the uptake experiment with sample 2, the possibility to describe the position of the liquid front with the modified Washburn's equation suggests that the water-glycerol case can be regarded as a single mixture with an average viscosity and surface tension, since Washburn's equation is only valid for a homogeneous liquid. 


\subsection{Water and water-glycerol sorption in $1100 \mathrm{~nm} \mathrm{Al}_{2} \mathrm{O}_{3}$.}

Finally, the effect of the average pore size of the $\mathrm{Al}_{2} \mathrm{O}_{3}$ samples is investigated. Therefore, we measured water and water-glycerol sorption in the $1100 \mathrm{~nm}$ porous $\mathrm{Al}_{2} \mathrm{O}_{3}$ samples. The water-glycerol sorption experiments for the $1100 \mathrm{~nm}$ case are included in fig. 8. This shows that the behavior of water and water-glycerol in these $\mathrm{Al}_{2} \mathrm{O}_{3}$ samples can be approximated using the modified Washburn's equation and implies the water-glycerol mixture moves into the $1100 \mathrm{~nm}$ samples as a homogeneous liquid.

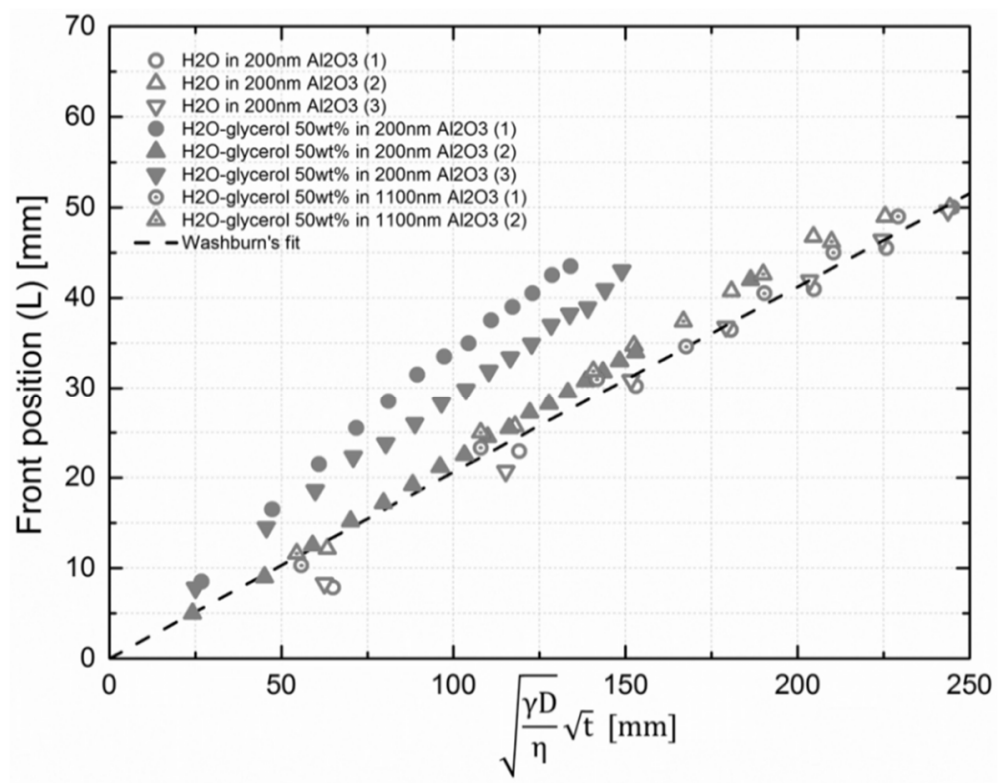

Figure 8: Front position as function of rescaled time for water-glycerol sorption in porous $\mathrm{Al}_{2} \mathrm{O}_{3}$.

\section{Discussion and conclusion}

In this study we investigated the behavior of water-glycerol mixtures penetrating in porous $\mathrm{Al}_{2} \mathrm{O}_{3}$ to determine how fast and how far each component penetrates. We found that the front speed of water and water-glycerol can be well described by the modified Washburn's equation and the front position as function of time can be predicted by this simple formula.

We found that the results of the liquid sorption experiments can be mapped on a single line by scaling with the liquid properties (viscosity and surface tension) and the properties of the porous material (average pore diameter). This indicates that water-glycerol $50 \mathrm{wt} \%$ can be regarded as a homogenous mixture with an average viscosity and surface tension, when penetrating into the $1100 \mathrm{~nm} \mathrm{Al} \mathrm{O}_{2}$ samples. 
This also implies that there is no liquid separation during the imbibition process.

In the case of water-glycerol sorption in the $200 \mathrm{~nm}$ porous $\mathrm{Al}_{2} \mathrm{O}_{3}$ samples we found that 3 samples from the same batch show different behavior. Although the sorption in all measurements still scales with liquid parameters and with the square root of time, the slope cannot be explained with reasonable values for the contact angle alone. Secondly we know that the tortuosity of the samples is very similar since the water sorption experiments on the same samples give comparable results. Next steps will address this issue.

The next step towards understanding the liquid penetration of water-based inks in paper will be to add particles to the penetrating liquid and to investigate how this influences the sorption behavior.

\section{Acknowledgements}

This research was carried out under project number M61.2.12454a in the framework of the Research Program of the Materials innovation institute (M2i) (www.m2i.nl). The authors thank Jef Noijen and Hans Dalderop for their technical support with the experiments. For the help in deciding the model system for inkpaper interaction we would like to thank Louis Saes and Herman Wijshoff of the R\&D department of Océ. Finally we would like to thank Peter Lipman for his help with the MIP measurements.

\section{References}

[1] Darcy, H., Les fontaines publiques de la ville de Dijon, Dalmont: Paris, p. $647,1856$.

[2] Richards, L.A., Capillary conduction of liquids through porous mediums, J. Appl. Phys., 1(5), pp. 318-333, 1931.

[3] Washburn, E., The dynamics of capillary flow, Phys. Rev., 17(3), pp. 273283, 1921.

[4] Sarkar, A.K, Georgiou, G. \& Sharma, M.M., Transport of bacteria in porous media: I. An experimental investigation, Biotechnol. Bioeng., 44(4), pp. 489-497, 1994.

[5] Blunt, M., Bijeljic, B., Dong, H. \& Gharbi, O., Pore-scale imaging and modelling, Adv. Water Res., 51, pp. 197-216, 2013.

[6] Szulczewski, M.L., Cueto-Felgueroso, L. \& R. Juanes, Scaling of capillary trapping in unstable two-phase flow: Application to $\mathrm{CO}_{2}$ sequestration in deep saline aquifers, Energy Procedia, 1(1), pp. 3421-3428, 2009.

[7] Wang, Z., Wang, C. \& Chen, K., Two-phase flow and transport in the air cathode of proton exchange membrane fuel cells, J. Power Sources, 94, pp. 40-50, 2001.

[8] Murdoch, A. \& Kowalski, S., On fluid-fluid coupling within porous media: a mixture theoretic approach based upon molecular considerations, Transp. porous media, 8, pp. 47-70, 1992. 
[9] Anderson, D.M., Imbibition of a liquid droplet on a deformable porous substrate, Phys. Fluids, 17(8), 2005.

[10] Bowen, R., Compressible porous media models by use of the theory of mixtures, Int. J. Eng. Sci., 20(6), pp. 697-735, 1982.

[11] Van Brakel, J., Pore space models for transport phenomena in porous media review and evaluation with special emphasis on capillary liquid transport, Powder Technol., 11, pp. 205-236, 1975.

[12] Juanes, R., Nonequilibrium effects in models of three-phase flow in porous media, Adv. Water Resour., 31(4), pp. 661-673, 2008.

[13] Blunt, M., Flow in porous media-pore-network models and multiphase flow, Curr. Opin. Colloid Interface Sci., 6(3), pp. 197-207, 2001.

[14] Sharma, M.M. \& Yortsos, Y.C., Transport of Particulate Suspensions in Porous Media: Model Formulation. AIChE J., 33(10), pp. 1636-1643, 1987.

[15] Nikan'shin, D. \& Nikiforov A., Modeling of transfer of particles of different size by a two-phase filtration flow, J. Eng. Phys, 73(3), pp. 497-500, 2000.

[16] Helmig, R., Niessner, J., Flemisch, B., Wolff, M. \& Fritz, J., Efficient modelling of flow and transport in porous media using multi-physics and multi-scale approaches, SRC SimTech, 27, pp. 1-34, 2010.

[17] Horgue, P., Augier, F., \& Duru, P., Experimental and numerical study of two-phase flows in arrays of cylinders, Chem. Eng., 102, pp. 335-345, 2013.

[18] Weisbrod, N., McGinnis, T., Rockhold, M.L., Niemet, M.R. \& Selker, J. S., Effective Darcy-scale contact angles in porous media imbibing solutions of various surface tensions, Water Resour. Res., 45(10), W00D39, 2009.

[19] Glass, R., Steenhuis, T. \& Parlange, J.-Y., Wetting front instability: 2. Experimental determination of relationships between system parameters and two-dimensional unstable flow field behavior in initially dry porous media, Water Resour. Res., 25(6), pp. 1195-1207, 2010.

[20] Stevar M.S.P. \& Vorobev, A., Dissolution Dynamics of Liquid/Liquid Binary Mixtures Within a Micromodel, Transp. Porous Media, 100(3), pp. 407-424, 2013.

[21] Davarzani, H. \& Marcoux, M., Experimental measurement of the effective diffusion and thermodiffusion coefficients for binary gas mixture in porous media, Chem. Eng., 65(18), pp. 5092-5104, 2010.

[22] Pel, L., Kopinga, K., \& Brocken, H., Moisture transport in porous building materials, Heron, 41(2), pp. 95-105, 1996.

[23] Reuvers, N.J.W., Huinink, H.P., Adan, O.C.G., Garcia, S.J. \& Mol, J.M.C. Water uptake in thin nylon 6 films as measured by electrochemical impedance spectroscopy and magnetic resonance imaging, Electrochim. Acta, 94, pp. 219-228, 2013.

[24] Merz, S., Pohlmeier, A., Vanderborght, J., van Dusschoten, D. \& Vereecken, H., Moisture profiles of the upper soil layer during evaporation monitored by NMR, Water Resour. Res., 50(6), pp. 5184-5195, 2014.

[25] Gombia, M. \& Bortolotti, V., Models of water imbibition in untreated and treated porous media validated by quantitative magnetic resonance imaging, J. Appl. Phys., 103(9), 094913, 2008. 
[26] Fridjonsson, E.O., Codd, S.L. \& Seymour, J.D., Application of PFG-NMR to Study the Impact of Colloidal Deposition on Hydrodynamic Dispersion in a Porous Medium, Transp. Porous Media, 103(1), pp. 117-130, 2014.

[27] Bell, J. \& Cameron, F., The flow of liquids through capillary spaces, J. Phys. Chem., 50(30), pp. 658-674, 1906.

[28] Blümich, B., NMR imaging of materials, Oxford University Press Inc.: New York, pp. 1-50, 2000.

[29] Hahn, E.L., Spin echoes, Phys. Rev., 80(4), pp. 580-594, 1950.

[30] Callaghan, P.T., Principles of buclear magnetic resonance microscopy, Oxford University Press Inc.: New York, pp. 25-92, 1991.

[31] Kopinga, K. \& Pel, L., One-dimensional scanning of moisture in porous materials with NMR, Rev. Sci. Instrum, 65, pp. 3673-3681, 1994.

[32] Bosart L.W. \& Snoddy, A.O., Specific Gravity of Glycerol, Ind. Eng. Chem., 20(12), pp. 1377-1379.

[33] Dorsey, N.E., Properties of Ordinary Water Substance, Reinhold: New York, p. 184, 1940.

[34] Muller, E., Sitzber: Akad. Wiss. Wien, Math.-Naturew. Klasse, Abt.IIa, pp. 133-147, 1924. 